CORRECTION

https://doi.org/10.1038/s41586-018-0723-9

\title{
Author Correction: Necroptosis microenvironment directs lineage commitment in liver cancer
}

Marco Seehawer, Florian Heinzmann, Luana D’Artista, Jule Harbig, Pierre-François Roux, Lisa Hoenicke, Hien Dang, Sabrina Klotz, Lucas Robinson, Grégory Doré, Nir Rozenblum, Tae-Won Kang, Rishabh Chawla, Thorsten Buch, Mihael Vucur, Mareike Roth, Johannes Zuber, Tom Luedde, Bence Sipos, Thomas Longerich, Mathias Heikenwälder, Xin Wei Wang, Oliver Bischof \& Lars Zender

Correction to: Nature https://doi.org/10.1038/s41586-018-0519-y, published online 12 September 2018.

In this Article, the pCaMIN transposon plasmid consisted of 'mouse $M y c$ and mouse Nras ${ }^{G 12 V}$ ' instead of 'mouse $M y c$ and human NRAS ${ }^{G 12 V}$, and the pCAMIA construct consisted of 'mouse $M y c$ and human $A K T 1$ ' instead of 'mouse $M y c$ and $A k t 1$ '; this has been corrected online throughout the Article. 